the hazard ratio for mortality for patients who received radiotherapy and 3 or 6 months shortterm androgen deprivation was $0.95(P=0.79)$ and $0.56(P=0.01)$, respectively. TTBF was a better predictor of this trial finding than PSADT. Both PSADT and TTBF satisfied all four Prentice criteria at multiple time-points ( $<12$ and $<15$ months for PSADT, and $<1.5,<2$ and $<2.5$ years for TTBF).

The use of PSADT and TTBF as surrogate end points for prostate-cancer-specific mortality needs to be validated in meta-analyses of clinical trial data. Use of these end points could substantially reduce the need for prolonged follow-up in clinical trials of prostate cancer therapy.

Original article Denham JW et al. (2008) Time to biochemical failure and prostate-specific antigen doubling time as surrogates for prostate cancer-specific mortality: evidence from the TROG 96.01 randomised controlled trial. Lancet Oncol 9: 1058-1068

\section{Linear mixed model helps to quantify PSA profile and predict disease progression}

Assessment of prostate-specific antigen (PSA) kinetics is often used to predict outcome after radiation therapy for localized prostate cancer. The decision to start salvage therapy is usually made before the development of symptomatic disease, and which PSA characteristics should be used for treatment decisions remains unclear. Proust-Lima and colleagues have developed an approach based on the relationships between prognostic factors, postradiotherapy PSA kinetics and clinical recurrence to provide an accurate picture of the risk of recurrence.

The authors analyzed 40,324 PSA measurements from 4,247 patients treated with radiation therapy. A linear mixed model was used to describe the pattern of PSA changes after radiotherapy. A Cox model was then used to determine the association between prognostic factors, post-treatment PSA behavior and time to recurrence.

Clinical recurrences were observed in $12.2 \%$ of the patients. Short-term declines in PSA levels after radiation were significantly associated with pretreatment PSA and tumor stage. High values of pretreatment PSA, tumor-stage and Gleason score were associated with steep subsequent increases in PSA. A high current PSA level and steep rate of PSA increase were both associated with a high risk of recurrence.

The authors conclude that a joint linear mixed model can be used to assess the PSA kinetics and risk of recurrence after radiation therapy for prostate cancer and have developed a risk calculator for others to use.

Original article Proust-Lima C et al. (2008) Determinants of change in prostate-specific antigen over time and its association with recurrence after external beam radiation therapy for prostate cancer in five large cohorts. Int J Radiat Oncol Biol Phys 72: 782-791

\section{Gene-expression profiles in fixed tissues correlate with survival in patients with HCC}

Identifying patients with hepatocellular carcinoma (HCC) who are at greatest risk of recurrence after potentially curative treatment is a challenge. Moreover, few techniques are available to assess genomic profiles as predictors of outcome in fixed tissue samples from patients. A recent study has shown that in patients with $\mathrm{HCC}$, genome-wide analysis of formalin-fixed, paraffin-embedded tissues (FPET) in tissue adjacent to the resected tumor correlates with survival.

Hoshida et al. developed a method to assess the gene profiles of over 6,000 genes in archived FPET tissues obtained from 106 Japanese patients treated with surgery for primary HCC (training set) and 234 European and American patients with HCC treated with surgery (validation set). Samples from $90 \%$ of the patients were of high enough quality for assessment, including those archived for over 24 years. No association between gene-expression profiles of the tumor tissue and tumor recurrence or clinical outcome was noted. The authors hypothesized that tissue surrounding the tumor might harbor a gene-expression signature that predicts recurrence. A 186-gene signature associated with survival was shown to correlate significantly with survival in tissue samples from 82 Japanese patients $(P=0.02)$. Furthermore, validation of this signature in an independent set of samples from 225 patients treated in the US and Europe was also significantly correlated with survival $(P=0.04)$.

This study showed that genome-wide expression profiling in FPET tissue is possible, 